KYUNGPOOK Math. J. 53(2013), 507-513

http://dx.doi.org/10.5666/KMJ.2013.53.4.507

\title{
Some New Application of Power Increasing Sequences
}

WaAd Sulaiman

Department of Computer Engineering, College of Engineering, University of Mosul, Mosul, Iraq

e-mail: waadsulaiman@hotmail.com

ABSTRACT. A general theorem for absolute summability using quasi $f$-power increasing sequence is obtained. This result generalized and improved the result of Bor and Özarslan [2].

\section{Introduction}

Let $\sum a_{n}$ be an infinitive series with the sequence of partial sums $\left(s_{n}\right)$. By $(C, \alpha)$ we mean the Cesàro matrix of order $\alpha$, and $\sigma_{n}^{\alpha}$ denotes the n-th term of the $(C, \alpha)$-transform of $\left(s_{n}\right)$, that is

$$
\sigma_{n}^{\alpha}=\frac{1}{A_{n}^{\alpha}} \sum_{v=0}^{n} A_{n-v}^{\alpha-1} s_{v} .
$$

A series $\sum a_{n}$ is said to be summable $|C, \alpha|_{k}, k \geq 1$, if

$$
\sum_{n=1}^{\infty} n^{k-1}\left|\sigma_{n}^{\alpha}-\sigma_{n-1}^{\alpha}\right|^{k}<\infty,
$$

and it is said to be summable $\varphi-|C, \alpha|_{k}, k \geq 1, \alpha>-1$ if (see Balc1 [1])

$$
\sum_{n=1}^{\infty}\left|\varphi_{n}\left(\sigma_{n}^{\alpha}-\sigma_{n-1}^{\alpha}\right)\right|^{k}<\infty,
$$

or equivalently

$$
\sum_{n=1}^{\infty} n^{-k}\left|\varphi_{n} t_{n}^{\alpha}\right|^{k}<\infty
$$

where

$$
t_{n}^{\alpha}=\frac{1}{A_{n}^{\alpha}} \sum_{v=0}^{n} A_{n-v}^{\alpha-1} v a_{v} .
$$

Received September 8, 2011; accepted February 14, 2012.

2010 Mathematics Subject Classification: 40F05, 40D25, 40 G99.

Key words and phrases: Absolute summability. 
In the special case when $\varphi_{n}=n^{1-1 / k}, n^{\delta+1-1 / k}(n \in N, k \geq 1), \varphi-|C, \alpha|_{k}$-summability reduces to $|C, \alpha|_{k}$-summability, $|C, \alpha, \delta|_{k}$-summability respectively. Let $\left(p_{n}\right)$ be a sequence of positive real numbers such that

$$
P_{n}=p_{0}+p_{1}+\ldots+p_{n} \rightarrow \infty \text { as } n \rightarrow \infty, P_{-1}=p_{-1}=0 .
$$

A series $\sum a_{n}$ is said to be summable $\left|N, p_{n}\right|_{k}, k \geq 1$, if

$$
\sum_{n=1}^{\infty} n^{k-1}\left|u_{n}-u_{n-1}\right|^{k}<\infty
$$

where

$$
u_{n}=\frac{1}{P_{n}} \sum_{n=1}^{\infty} p_{n-v} s_{v} .
$$

For $k=1,\left|N, p_{n}\right|_{k}$-summability reduces to $\left|N, p_{n}\right|$-summability, and for $p_{n}=A_{n}^{\alpha}$, $\left|N, p_{n}\right|_{k}$-summability reduces to $|C, \alpha|_{k}$-summability. By $M$ we denote the set of sequences $p=\left(p_{n}\right)$ satisfying

$$
\frac{p_{n+1}}{p_{n}} \leq \frac{p_{n+2}}{p_{n+1}} \leq 1, \quad p_{n}>0, \quad n=0,1, \ldots .
$$

It is known that (see Das [4]) for $p \in M, k=1,(1.7)$ holds if and only if

$$
\sum_{n=1}^{\infty} \frac{1}{n P_{n}}\left|\sum_{v=1}^{n} p_{n-v} v a_{v}\right|<\infty .
$$

For $p \in M$, the series $\sum a_{n}$ is summable $\left|N, p_{n}\right|_{k}, k \geq 1$, if (see Sulaiman [7])

$$
\sum_{n=1}^{\infty} \frac{1}{n P_{n}^{k}}\left|\sum_{v=1}^{n} p_{n-v} v a_{v}\right|^{k}<\infty .
$$

Here we give the following definition: For $p \in M$, the series $\sum a_{n}$ is summable $\varphi-\left|N, p_{n}\right|_{k}, k \geq 1$, if

$$
\sum_{n=1}^{\infty} n^{-k}\left|\varphi_{n} \Phi_{n}\right|^{k}<\infty
$$

where

$$
\Phi_{n}=\frac{1}{P_{n}} \sum_{v=1}^{n} p_{n-v} v a_{v} .
$$


A positive sequence $a=\left(a_{n}\right)$ is said to be quasi $\beta$-power increasing sequence if there exists a constant $K=K(\beta, a) \geq 1$ such that (see Leindler [5])

$$
K n^{\beta} a_{n} \geq m^{\beta} a_{m} \quad(n \geq m \geq 1, n, m \in N) .
$$

A positive sequence $\left(a_{n}\right)$ is said to be quasi $f$-power increasing sequence if there exists a constant $K=K(f, a) \geq 1$ such that (see Sulaiman [8])

$$
K f_{n} a_{n} \geq f_{m} a_{m} \quad(n \geq m \geq 1, n, m \in N) .
$$

where $f=\left(f_{n}\right), f=n^{\beta}(\log n)^{\gamma}, 0<\beta<1, \gamma \geq 0$. Recently, Bor and Özarslan [2] presented the following theorem

Theorem 1.1. Let $\left(X_{n}\right)$ be a quasi- $\beta$-power increasing sequence for some $\beta(0<\beta<1)$. Suppose also that there exist sequences $\left(\beta_{n}\right)$ and $\left(\lambda_{n}\right) \in B V_{0}$ such that

$$
\left|\Delta \lambda_{n}\right| \leq \beta_{n}
$$

$$
\beta_{n} \rightarrow \infty, \quad(n \rightarrow \infty)
$$

and

$$
\sum_{n=1}^{\infty} n\left|\Delta \beta_{n}\right| X_{n}<\infty
$$

If there exists an $\epsilon>0$ such that the sequence $\left(n^{\epsilon-k}\left|\varphi_{n}\right|^{k}\right)$ is non-increasing and if the sequence $\left(\omega_{n}^{\alpha}\right)$ is defined by

$$
\omega_{n}^{\alpha}=\left\{\begin{array}{l}
\left|t_{n}^{\alpha}\right|, \quad \alpha=1 \\
\max _{1 \leq v \leq n}\left\{\left|t_{v}^{\alpha}\right|\right\}, 0<\alpha<1
\end{array}\right.
$$

satisfying the following condition:

$$
\sum_{n=1}^{m} n^{-k}\left(\left|\varphi_{n}\right| \omega_{n}^{\alpha}\right)^{k}=O\left(X_{m}\right), \quad(m \rightarrow \infty)
$$

then the series $\sum a_{n} \lambda_{n}$ is summable $\varphi-|C, \alpha|_{k}, k \geq 1,0<\alpha \leq 1, k \alpha+\epsilon>1$.

\section{Lemmas}

In this section, the following lemmas are needed for our aim. 
Lemma 2.1. If $0 \leq m \leq n, p_{n-v} / p_{m-v}$ is decreasing with respect to $v$, then

$$
\left|\sum_{v=0}^{m} p_{n-v} s_{v}\right| \leq \max _{0 \leq \mu \leq m}\left|\sum_{v=0}^{\mu} p_{\mu-v} s_{v}\right| .
$$

Proof. The proof is similar to that given in [3].

Lemma 2.2. Condition (1.14) - (1.16) implies

$$
n \beta_{n} X_{n}=O(1), n \rightarrow \infty,
$$

$$
\sum_{n=1}^{\infty} \beta_{n} X_{n}<\infty
$$

Proof. The proof is similar to that given in [8].

\section{Results}

The following is our main result.

Theorem 3.1. Let $\left(X_{n}\right)$ be a quasi-f-power increasing sequence, $f=\left(f_{n}\right), f_{n}=$ $n^{\beta}(\log n)^{\gamma}, 0<\beta<1, \gamma \geq 0$, such that the sequences $\left(X_{n}\right)$ and $\left(\lambda_{n}\right)$ satisfying conditions $(1.14)-(1.17)$, and

$$
\begin{gathered}
\sum_{n=1}^{m} \frac{1}{n^{k} X_{n}^{k-1}}\left(\left|\varphi_{n}\right| \omega_{n}\right)^{k}=O\left(X_{m}\right), \quad(m \rightarrow \infty), \\
\sum_{n=v+1}^{m+1} \frac{\left|\varphi_{n}\right|^{k}}{n^{k} P_{n}^{k}}=O\left(\frac{\left|\varphi_{v}\right|^{k}}{v^{k-1} P_{v}^{k}}\right)
\end{gathered}
$$

where

$$
\omega_{n}=\left\{\begin{array}{l}
\Phi_{n}, \quad v=n \\
\max \left\{\Phi_{v}\right\}, 1 \leq v \leq n .
\end{array}\right.
$$

Then the series $\sum a_{n} \lambda_{n}$ is summable $\varphi-\left|N, p_{n}\right|_{k}, k \geq 1$.

Proof. Let $T_{n}$ be n-th $\left(N, p_{n}\right)$-mean of the sequence $\left(n a_{n} \lambda_{n}\right)$. Then, we have

$$
T_{n}=\frac{1}{P_{n}} \sum_{v=1}^{n} v a_{v} p_{n-v} \lambda_{v}
$$


We have, via Abel's transformation

$$
\begin{aligned}
\left|T_{n}\right| & =\frac{1}{P_{n}}\left|\sum_{v=1}^{n} v a_{v} p_{n-v} \lambda_{v}\right| \\
& =\frac{1}{P_{n}}\left|\sum_{v=1}^{n-1}\left(\sum_{r=1}^{v} r p_{n-r} a_{r}\right) \Delta \lambda_{v}+\lambda_{n} \sum_{v=1}^{n} v p_{n-v} a_{v}\right| \\
& \leq \frac{1}{P_{n}} \sum_{v=0}^{n-1} P_{v}\left|\omega_{v}\right|\left|\Delta \lambda_{v}\right|+\left|\lambda_{n}\right|\left|\omega_{n}\right| \\
& =T_{n 1}+T_{n 2} .
\end{aligned}
$$

Since $\left|T_{n 1}+T_{n 2}\right|^{k} \leq 2^{k}\left(\left|T_{n 1}\right|^{k}+\left|T_{n 2}\right|^{k}\right)$, then in order to complete the proof, it is sufficient to show that

$$
\sum_{n=1}^{\infty} n^{-k}\left|\varphi_{n} T_{n j}\right|^{k}<\infty, \quad j=1,2 .
$$

Applying Hölder's inequality,

$$
\begin{aligned}
\sum_{n=2}^{m+1} n^{-k}\left|\varphi_{n} T_{n 1}\right|^{k}= & \sum_{n=2}^{m+1} \frac{\left|\varphi_{n}\right|^{k}}{n^{k} P_{n}^{k}}\left|\sum_{v=1}^{n-1} P_{v}\right| \omega_{v}|| \Delta \lambda_{v}||^{k} \\
& \leq \sum_{n=2}^{m+1} \frac{\left|\varphi_{n}\right|^{k}}{n^{k} P_{n}^{k}} \sum_{v=1}^{n-1} P_{v}^{k}\left|\omega_{v}\right|^{k} \beta_{v} X_{v}^{1-k}\left(\sum_{v=1}^{n-1} X_{v} \beta_{v}\right)^{k-1} \\
= & O(1) \sum_{n=2}^{m+1} \frac{\left|\varphi_{n}\right|^{k}}{n^{k} P_{n}^{k}} \sum_{v=1}^{n-1} P_{v}^{k}\left|\omega_{v}\right|^{k} \beta_{v} X_{v}^{1-k} \\
= & O(1) \sum_{v=1}^{m} P_{v}^{k}\left|\omega_{v}\right|^{k} \beta_{v} X_{v}^{1-k} \sum_{n=v+1}^{m+1} \frac{\left|\varphi_{n}\right|^{k}}{n^{k} P_{n}^{k}} \\
= & O(1) \sum_{v=1}^{m} \frac{v}{v^{k} X_{v}^{k-1}\left|\varphi_{v}\right|^{k}\left|\omega_{v}\right|^{k} \beta_{v}} \\
= & O(1) \sum_{v=1}^{m}\left(\sum_{r=1}^{v} \frac{\left|\varphi_{r}\right|^{k}\left|\omega_{r}\right|^{k}}{r^{k} X_{r}^{k-1}}\right)^{\Delta\left(v \beta_{v}\right)} \\
& +O(1) m \beta_{m} \sum_{v=1}^{m} \frac{1}{v^{k} X_{v}^{k-1}}\left|\varphi_{v}\right|^{k}\left|\omega_{v}\right|^{k} \\
= & O(1) \sum_{v=1}^{m} \beta_{v} X_{v}+O(1) \sum_{v=1}^{m} v\left|\Delta \beta_{v}\right| X_{v}+O(1) m \beta_{m} X_{m} \\
= & O(1) .
\end{aligned}
$$




$$
\begin{aligned}
\sum_{n=1}^{m} n^{-k}\left|\varphi_{n} T_{n 2}\right|^{k} & =\sum_{n=1}^{m} \frac{\left|\varphi_{n}\right|^{k}}{n^{k}}\left|\lambda_{n}\right|^{k}\left|\omega_{n}\right|^{k} \\
& =\sum_{n=1}^{m} \frac{\left|\varphi_{n}\right|^{k}}{n^{k} X_{n}^{k-1}}\left|\lambda_{n}\right|\left|\omega_{n}\right|^{k}\left(\left|\lambda_{n}\right| X_{n}\right)^{k-1} \\
& =O(1) \sum_{n=1}^{m} \frac{\left|\varphi_{n}\right|^{k}\left|\omega_{n}\right|^{k}}{n^{k} X_{n}^{k-1}}\left|\lambda_{n}\right| \\
& =O(1) \sum_{n=1}^{m}\left(\sum_{v=1}^{n} \frac{\left|\varphi_{v}\right|^{k}\left|\omega_{v}\right|^{k}}{v^{k} X_{v}^{k-1}}\right) \Delta\left|\lambda_{n}\right|+O(1)\left|\lambda_{m}\right| \sum_{n=1}^{m} \frac{\left|\varphi_{n}\right|^{k}\left|\omega_{n}\right|^{k}}{n^{k} X_{n}^{k-1}} \\
& =O(1) \sum_{n=1}^{m}\left|\Delta \lambda_{n}\right| X_{n}+O(1)\left|\lambda_{m}\right| X_{m} \\
& =O(1) \sum_{n=1}^{m} \beta_{n} X_{n}+O(1)\left|\lambda_{m}\right| X_{m} \\
& =O(1) .
\end{aligned}
$$

Remark 1. (1) It may be mentioned that an improvement to Theorem 1.1 follows from Theorem 3.1 by putting $p_{n}=A_{n}^{\alpha-1}$, provided from some $\epsilon>0,\left(n^{\epsilon-k}\left|\varphi_{n}\right|^{k}\right)$ is non-increasing, and $k \alpha+\epsilon>1$.

(2) One advantage of condition (3.1) is that we have no loss of power of $\left|\lambda_{n}\right|$ through estimation such as by having $\left|\lambda_{n}\right|^{k-1}=O(1)$.

Remark 2. Condition (3.1) does not imply condition (1.19) .

Proof.

$$
\begin{aligned}
\sum_{n=1}^{m} n^{-k}\left(\left|\varphi_{n}\right| \omega_{n}^{\alpha}\right)^{k} & =\sum_{n=1}^{m} \frac{1}{n^{k} X_{n}^{k-1}}\left(\left|\varphi_{n}\right| \omega_{n}^{\alpha}\right)^{k} X_{n}^{k-1} \\
& =O\left(X_{m}^{k-1}\right) \sum_{n=1}^{m} \frac{1}{n^{k} X_{n}^{k-1}}\left(\left|\varphi_{n}\right| \omega_{n}^{\alpha}\right)^{k} \\
& =O\left(X_{m}^{k}\right) \\
& \neq O\left(X_{m}\right), \text { for } k>1 .
\end{aligned}
$$




\section{References}

[1] M. Balci, Absolute $\varphi$-summability factors, Comm. Fac. Sci. Univ. Ankara Ser. $\mathrm{A}_{1}$, $\mathbf{2 9}(8)(1980), 63-80$.

[2] H. Bor and H. S. Özarslan, A study on quasi power increasing sequences, Rocky Mountain J. Math., 38(2008), 801-807.

[3] L. S. Bosanquet, A mean value theorem, J. London Math. Soc., 16(1941), 146-148.

[4] G. Das, Tauberian theorems for absolute Nörlund summability, Proc. London Math. Soc., 19(1969), 457-484.

[5] L. Leindler, A new application of quasi power increasing sequences. Publ. Math. D., 58(2001), 791-796.

[6] W. T. Sulaiman, Relations on some summability methods, Proc. Amer. Math. Soc., 118(1993), 1139-1245.

[7] W. T. Sulaiman, A general theorem covering many absolute summability methods, Collequium Marhematicum, 80(1999), 245-252.

[8] W. T. Sulaiman, Extensions on absolute summability factors of infinite series, J. Math. Anal. App., 322(2006), 1224-1230. 